\title{
Sulforaphane reduces YAP/ $\Delta$ Np63 $\alpha$ signaling to reduce cancer stem cell survival and tumor formation
}

\author{
Matthew L. Fisher ${ }^{1}$, Nicholas Ciavattone ${ }^{1}$, Daniel Grun ${ }^{1}$, Gautam Adhikary ${ }^{1}$ and \\ Richard L. Eckert ${ }^{1,2,3,4}$ \\ ${ }^{1}$ Department of Biochemistry and Molecular Biology, University of Maryland School of Medicine, Baltimore, Maryland, USA \\ ${ }^{2}$ Department of Dermatology, University of Maryland School of Medicine, Baltimore, Maryland, USA \\ ${ }^{3}$ Department of Reproductive Biology, University of Maryland School of Medicine, Baltimore, Maryland, USA \\ ${ }^{4}$ The Marlene and Stewart Greenebaum Comprehensive Cancer Center, University of Maryland School of Medicine, Baltimore, \\ Maryland, USA \\ Correspondence to: Richard L. Eckert, email: reckert@umaryland.edu
}

Keywords: YAP, TAZ, hippo signaling, $\triangle \mathrm{Np} 63 \alpha$, sulforaphane

Received: July 06, $2017 \quad$ Accepted: July 31, 2017

Published: August 27, 2017

Copyright: Fisher et al. This is an open-access article distributed under the terms of the Creative Commons Attribution License 3.0 (CC BY 3.0), which permits unrestricted use, distribution, and reproduction in any medium, provided the original author and source are credited.

\section{ABSTRACT}

Epidermal squamous cell carcinoma (SCC) is among the most common cancers. SCC can be treated by surgical excision, but recurrence of therapy-resistant disease is a major problem. We recently showed that YAP1, the Hippo signaling transcription adaptor protein, and $\Delta \mathrm{Np} 63 \alpha$, a key epidermal stem cell survival protein, form a complex to drive epidermal cancer stem cell survival. In the present study, we demonstrate that YAP1 and $\Delta \mathrm{Np} 63 \alpha$ are important sulforaphane cancer prevention targets. We show that sulforaphane treatment increases YAP1 phosphorylation and proteolytic degradation. The loss of YAP1 is associated with a reduction in $\triangle N p 63 \alpha$ level and a reduction in ECS cell survival, spheroid formation, invasion and migration. Loss of YAP1 and $\triangle N$ p $63 \alpha$ is mediated by the proteasome and can be inhibited by lactacystin treatment. YAP1 or $\triangle \mathrm{Np} 63 \alpha$ knockdown replicates the responses to sulforaphane, and restoration of YAP1 or $\triangle N p 63 \alpha$ antagonizes sulforaphane action. Sulforaphane suppresses ECS cell tumor formation and this is associated with reduced levels of YAP1 and $\triangle N p 63 \alpha$. These studies suggest that YAP1 and $\triangle N p 63 \alpha$ may be important sulforaphane cancer preventive targets in epidermal squamous cell carcinoma.

\section{INTRODUCTION}

Epidermal squamous cell carcinoma is an extremely prevalent disease that is caused by skin exposure to various mutagens including UV irradiation [1]. It is treated by surgery, but the recurrence rate approaches $10 \%$ and the recurring tumors are aggressive and therapy resistant [1]. Increasing evidence suggests that cancer stem cells have a central role in facilitating tumor growth in squamous cell carcinoma and are important therapy targets $[2,3]$. Epidermal squamous cell carcinoma cancer stem cells (ECS cells) express stem cell markers, form aggressive and highly vascularized tumors, and display enhanced migratory and invasive potential [2]. Various proteins have been identified as associated with enhanced ECS cell survival, migration, and tumor formation [2-6]; however, the mechanisms that drive ECS cell survival are not well understood. We recently showed that LATS1, YAP1 and $\Delta \mathrm{Np} 63 \alpha$ comprise an important survival cascade in ECS cells [7]. Based on these studies, we proposed that suppression of LATS1 (Hippo) signaling leads to enhanced nuclear accumulation of YAP1 which forms a complex with and stabilizes $\triangle \mathrm{Np} 63 \alpha$ to enhance ECS cell survival [7]. Hippo signaling is a centrally important cascade that controls organ growth and limits organ size during development [8]. Large Tumor Suppressor 1 (LATS1), a serine/threonine kinase, is a key regulator in the Hippo signaling cascade [9]. Reduced LATS1 kinase activity is associated with enhanced cell proliferation [9], and LATS1 activity is often constitutively reduced in cancer cells [9]. 
LATS1 reduces cell proliferation by phosphorylating the pro-proliferation/survival transcription adaptor proteins, YAP1 and TAZ, resulting in their movement to the cytoplasm and subsequent degradation [9]. In contrast, non-phosphorylated YAP1 and TAZ interact in the nucleus to stimulate cell survival and proliferation $[10,11]$. YAP1 is overexpressed in many cancers [9] and YAP1 activity is associated with enhanced stem cell survival in epidermis and other tissues [10-12].

$\Delta \mathrm{Np} 63 \alpha$ is a key member of the p63 family of proteins that control epithelial stem cell status and fate $[13,14]$. Studies in mouse epidermis identify $\Delta \mathrm{Np} 63 \alpha$ as a key controller of differentiation [13-16]. The function of p63 in epithelial development was shown in p63 knockout mice where the newborn mice die due to an epidermal barrier defect [15]. $\Delta \mathrm{Np} 63 \alpha$ is the primary p63 form expressed in squamous epithelial tissues [17] and $\Delta \mathrm{Np} 63 \alpha$ overexpression is a frequent event in squamous cell carcinoma [18].

Sulforaphane, 1-isothiocyanato-4-(methylsulfinyl) butane, is a natural isothiocyanate cancer preventive agent derived from broccoli and other cruciferous vegetables [19]. SFN has several desirable properties as a cancer prevention agent, as it is highly bioavailable in blood and tissues, is effective at suppressing tumor growth, and has no known side effects [20-23]. SFN has been shown to inhibit cancer development in various tissues [24-28] including epidermis [6, 29-32], but the molecular mechanism of action is not well understood.

As the LATS1, YAP $1, \Delta \mathrm{Np} 63 \alpha$ cascade is a potent driver of cancer stem cell survival [7], we decided to determine whether SFN can suppress activity in this cascade as a mechanism to suppress ECS cell survival. Our studies show that SFN treatment increases YAP1 phosphorylation and degradation, reduces $\triangle \mathrm{Np} 63 \alpha$ levels and reduces ECS cell survival, spheroid formation, invasion, migration and tumor formation.

\section{RESULTS}

\section{SFN impacts YAP1 signaling}

A small population of squamous cell carcinoma cells $(0.15 \%)$ survive and grow as spheroids in non-attached conditions, and these ECS cells display elevated levels of epidermal and embryonic stem cell markers, and enhanced ability to invade matrigel and migrate [2, 4]. Moreover, ECS cells form highly aggressive and vascularized tumors as compared to non-stem cancer cells [2,3]. In the present study we examine the impact of SFN treatment on ECS cells $[6,32]$. SFN treatment of ECS cell spheroid cultures reduces spheroid formation and enhances spheroid fragmentation (Figure 1A) and also suppresses ECS cell matrigel invasion (Figure 1B) and migration on plastic
(Figure 1C). We recently showed that a signaling cascade that involves YAP1 and $\Delta$ Np63 $\alpha$ plays an important role in ECS cell survival [7]. We therefore assessed the impact of SFN on the YAP1, TAZ, $\triangle \mathrm{Np} 63 \alpha$ and TEAD transcription factors. TEAD factors comprise a family of four transcription factors that are frequent targets of the YAP1 and TAZ transcriptional adaptors [9]. As shown in Figure 1D, SFN treatment reduces YAP1 and increases YAP1- $P$, and this is associated with reduced $\Delta \mathrm{Np} 63 \alpha$. In contrast, TAZ, TAZ- $P$ and TEAD levels are not altered.

The finding that YAP1 phosphorylation is altered by SFN treatment prompted us to examine the impact of YAP1 knockdown on ECS cell survival. Figure 1E/1F/1G shows that YAP1 knockdown reduces spheroid formation, matrigel invasion and migration. Figure $1 \mathrm{H}$ confirms YAP1-siRNA dependent YAP1 knockdown and loss of $\triangle \mathrm{Np} 63 \alpha$, and confirm no change in TAZ, TAZ-P or TEAD factor level. To confirm that YAP1 is a relevant SFN target, we examined the impact of constitutivelyactive YAP1 expression on SFN suppression of ECS cell spheroid formation and invasion. Figure 1I/1J shows that YAP(S127A) expression partially reverses SFN suppression of spheroid formation and invasion, confirming YAP1 loss is essential for SFN action. Figure $1 \mathrm{~K}$ shows that $\mathrm{YAP}(\mathrm{S} 127 \mathrm{~A})$ expression is associated with increased $\triangle \mathrm{Np} 63 \alpha$ expression, which is consistent with a role for YAP1 in stabilizing $\Delta \mathrm{Np} 63 \alpha$ [7].

\section{Role of $\Delta \mathrm{Np} 63 \alpha$}

We have reported that YAP1 acts to maintain $\Delta \mathrm{Np} 63 \alpha$ level and that $\Delta \mathrm{Np} 63 \alpha$ is required for ECS cell survival, spheroid formation and invasion [7]. Figure 1D shows that SFN treatment reduces $\Delta \mathrm{Np} 63 \alpha$ level. To determine whether loss of $\Delta \mathrm{Np} 63 \alpha$ is required for SFN action, we monitored the impact of $\Delta \mathrm{Np} 63 \alpha$ knockdown on ECS cell function, and determined that $\Delta \mathrm{Np} 63 \alpha$ overexpression can reverse SFN action. Figure $2 \mathrm{~A} / 2 \mathrm{~B} / 2 \mathrm{C}$ shows that loss of $\Delta \mathrm{Np} 63 \alpha$ reduces ECS cell spheroid formation, invasion and migration. Figure 2D/2E shows that SFN treatment reduces ECS cell spheroid formation and invasion and that these changes are reversed by $\Delta \mathrm{Np} 63 \alpha$ overexpression. Figure $2 \mathrm{~F}$ confirms overexpression of $\Delta \mathrm{Np} 63 \alpha$ and expression vector treated cells and shows that increasing $\Delta$ Np $63 \alpha$ does not impact YAP1 level.

To understand the mechanism of $\Delta \mathrm{Np} 63 \alpha$ reduction, we monitored the impact of SFN treatment on $\triangle \mathrm{Np} 63 \alpha \mathrm{mRNA}$ and found no change (Figure $3 \mathrm{~A}$ ). We then examined the role of the proteasome. ECS cells were treated with SFN in the presence or absence of lactacystin, a proteasome inhibitor. Figure 3B shows that SFN treatment reduces $\Delta \mathrm{Np} 63 \alpha$ level and that this is reversed by co-treatment with lactacystin. Moreover, SFN treatment is associated with enhanced ubiquitination of $\Delta \mathrm{Np} 63 \alpha$, which is consistent with proteasome-associated 

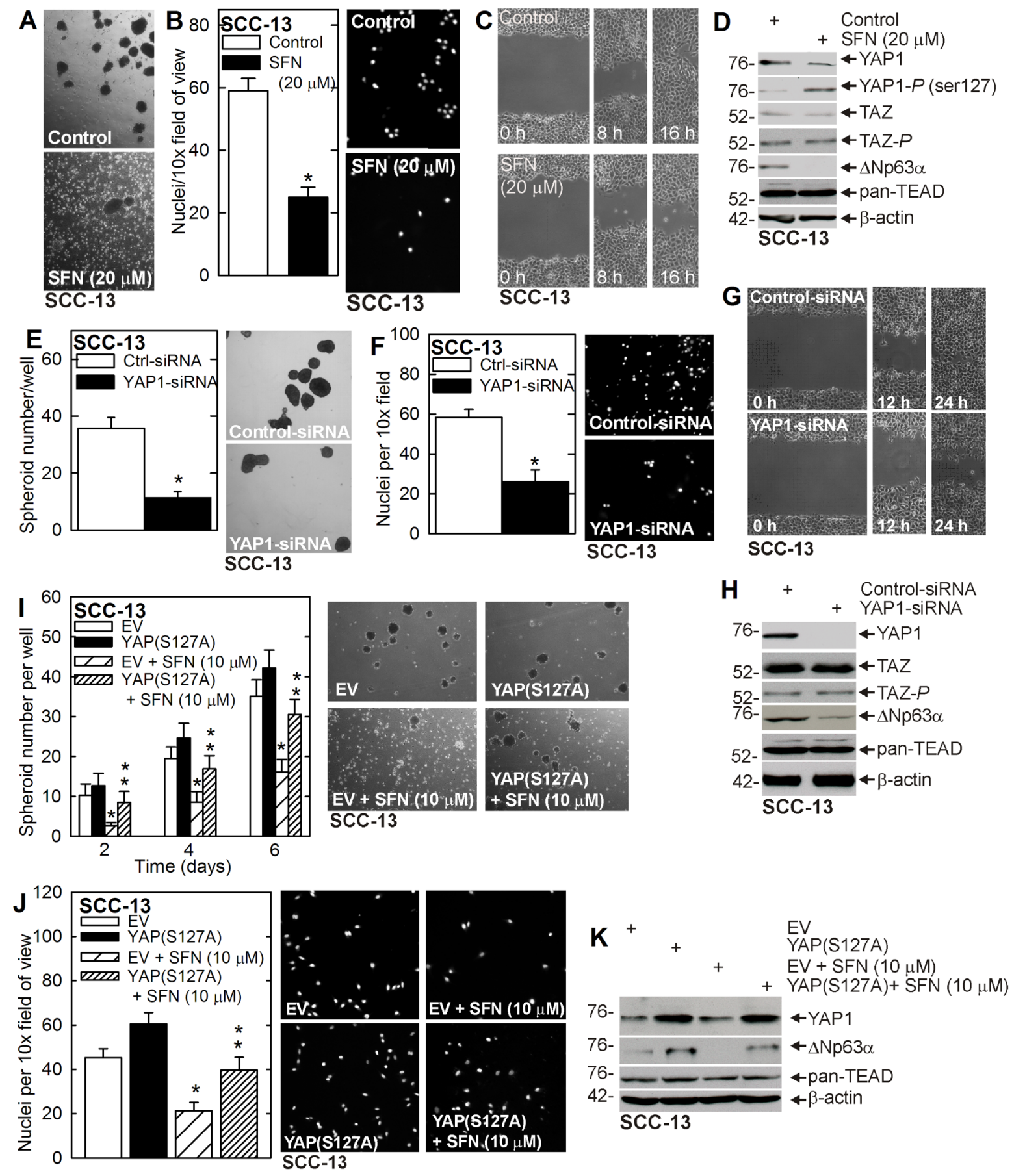

Figure 1: Sulforaphane targets YAP1/ $\triangle \mathrm{Np63 \alpha}$ to suppress ECS cell phenotype. A. B. C. ECS cells were grown for $8 \mathrm{~d}$ as spheroids and treated with 0 or $20 \mu \mathrm{M} \mathrm{SFN}$ for $48 \mathrm{~h}$ before image acquisition. ECS cells were seeded on a matrigel-coated membrane in a Millicell chamber for invasion assay and then treated with 0 or $20 \mu \mathrm{M}$ SFN for $20 \mathrm{~h}$. ECS cells were plated as high density confluent monolayers for wound closure assay in the presence of 0 or $20 \mu \mathrm{M}$ SFN. The values are mean $\pm \mathrm{SEM}$ and the asterisks indicate a significant reduction $(n=3, p<0.005)$. D. SFN treatment reduces YAP1, increases YAP1- $P$ and reduces $\Delta$ Np63 $\alpha$. Cells were grown as spheroids for 8 $\mathrm{d}$, treated with 0 or $20 \mu \mathrm{M} \mathrm{SFN}$ for $48 \mathrm{~h}$ and lysates were collected for immunoblot. E. F. G. SCC-13 cells were electroporated with controlor YAP1-siRNA and plated for spheroid formation, invasion and migration assay. The values are mean \pm SEM and the asterisks indicate a significant reduction $(n=3, p<0.005)$. H. YAP1-siRNA treatment reduces YAP1 and $\Delta$ Np63 $\alpha$ level, but does not impact TAZ or TEAD levels. I. J. SCC-13 cells, electroporated with empty vector (EV) or YAP(S127A), were seeded for spheroid growth or invasion assay in the presence of 0 or $10 \mu \mathrm{M}$ SFN. Spheroid number was monitored at $6 \mathrm{~d}$. The single asterisk indicates a significant reduction in SFN treated as compared to untreated control cultures. The double asterisks indicate a significant increase as compared to the SFN treated group $(n=3, p$ $<0.01)$. K. Immunoblot of extracts prepared from $5 \mathrm{~d}$ spheroid cultures (panel I). 

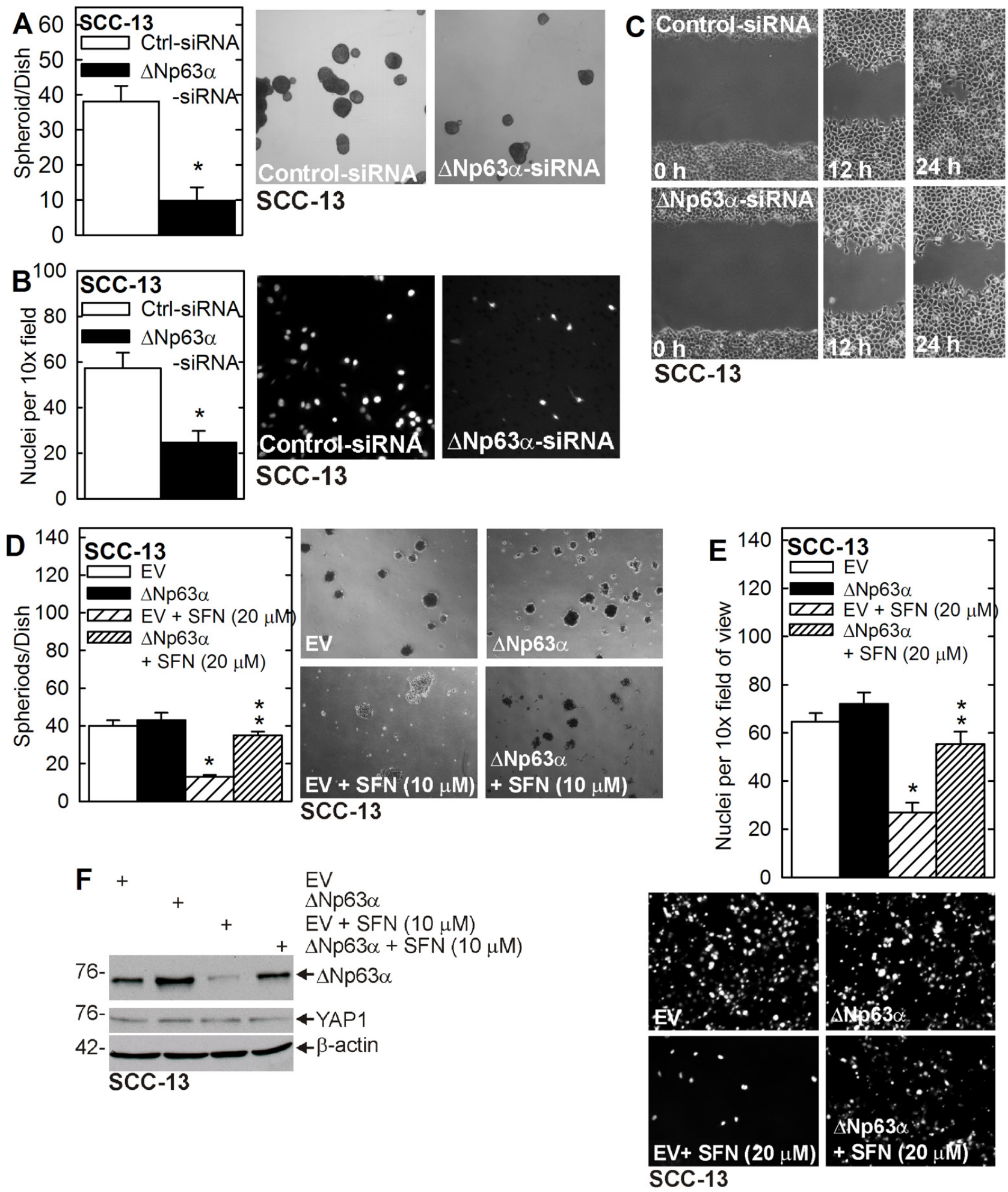

Figure 2: $\Delta$ Np63a drives the ECS cell phenotype. A. B. C. SCC-13 cells were double electroporated with control- or $\Delta$ Np63 $\alpha$ siRNA and seeded for spheroid formation, invasion and migration assay. The values are mean $\pm \mathrm{SEM}$ and the asterisks indicate a significant reduction $(n=3, p<0.01)$. D. E. ECS cells were electroporated with empty vector (EV) or $\Delta \mathrm{Np} 63 \alpha$ expression vector, and seeded for spheroid formation and invasion assays in the presence of 0 or $20 \mu \mathrm{M}$ SFN. The single asterisk indicates a significant reduction in SFN treated as compared to untreated control cultures. The double asterisks indicate a significant increase as compared to the SFN treated group $(n=3, p<0.01)$. F. Cells, treated as indicated, were grown in non-attached conditions for 5 days and lysates were prepared for immunoblot. 
degradation (Figure 3C). Thus, SFN stimulated $\Delta \mathrm{Np} 63 \alpha$ turnover is proteasome-mediated. Figure $3 \mathrm{D}$ indicates that most of cellular $\Delta \mathrm{Np} 63 \alpha$ is in the nucleus, as is $50 \%$ of YAP1, and that the nuclear level of both proteins is reduced by treatment with SFN.

\section{SFN impact on tumor formation}

We next determined the impact of SFN treatment on tumor formation. Figure 3E shows that SFN treatment produces a dose-dependent reduction in tumor formation that is optimal at 0.5 to 1 micromoles/dose. Figure
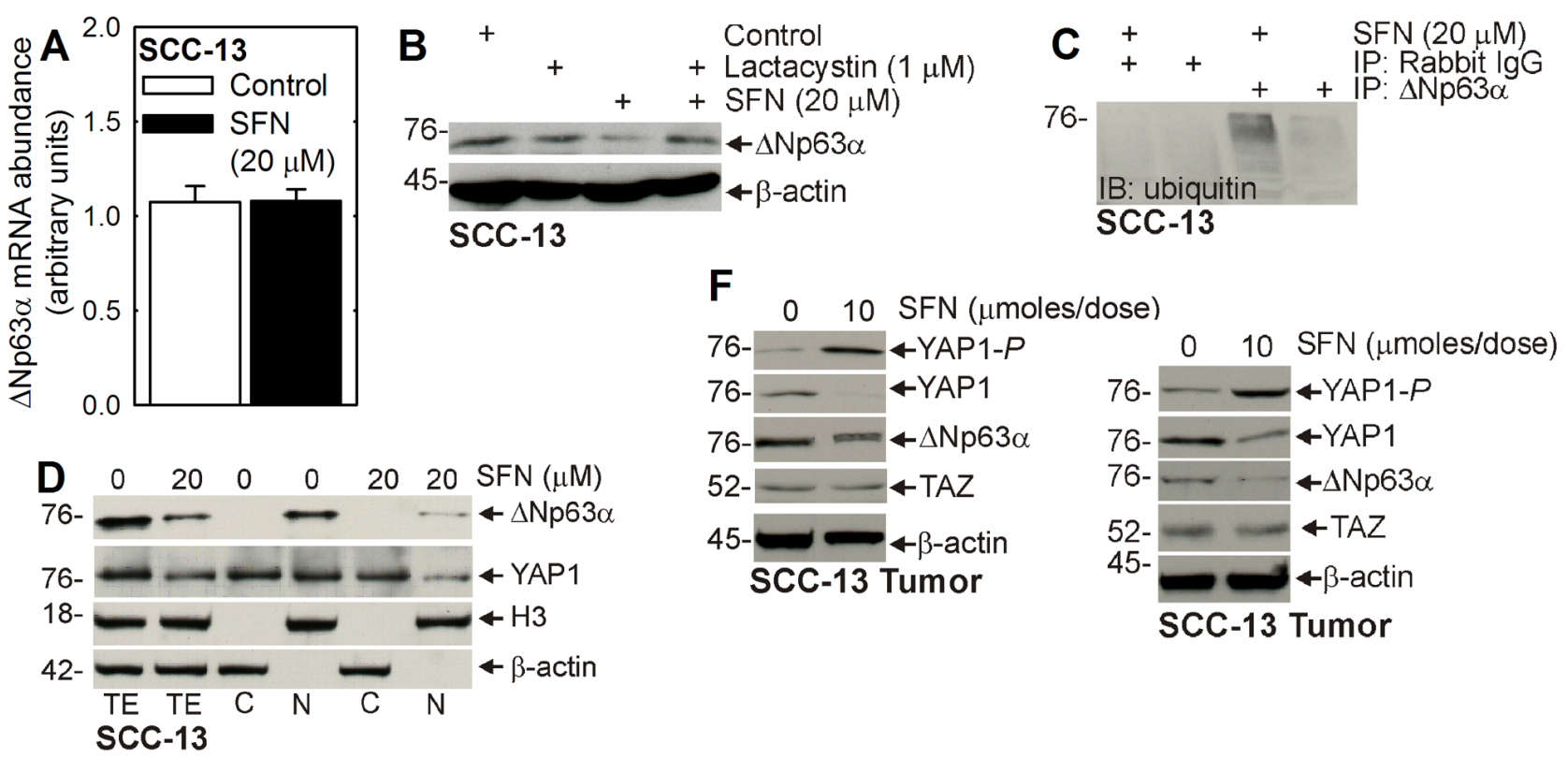

SCC-13

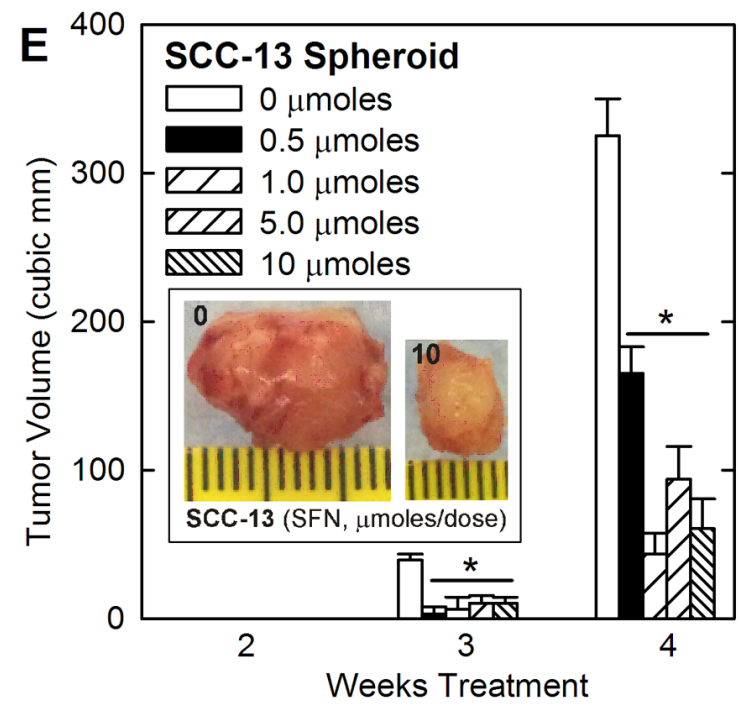

Figure 3: SFN induced proteasome-dependent loss of $\Delta \mathbf{N p 6 3 \alpha}$. A. ECS cells were treated with 0 or $20 \mu \mathrm{M}$ SFN for $48 \mathrm{~h}$ and extracts were prepared for assay of $\triangle \mathrm{Np} 63 \alpha$ mRNA by qRT-PCR. B. ECS cells were pre-treated with $1 \mu \mathrm{M}$ lactacystin for $1 \mathrm{~h}$, prior to the addition of $20 \mu \mathrm{M}$ SFN for $24 \mathrm{~h}$. C. ECS cells were treated with SFN for $48 \mathrm{~h}$ and lysates immunoprecipitated with anti- $\Delta \mathrm{Np} 63 \alpha$ for anti-ubiquitin immunoblot. D. Equal cell equivalents of total (TE), nuclear (N), and cytosolic (C) extract, prepared from control or $48 \mathrm{~h}$ SFN treated ECS cells, were electrophoresed for immunoblot detection of $\Delta \mathrm{Np} 63 \alpha$, YAP1, histone 3 (nuclear marker) and $\beta$-actin (cytoplasmic marker). E. ECS cells (100,000 cells derived from SCC-13) were injected into each front flank in NSG mice. Beginning at 1 d post-injection, SFN was delivered by gavage, three times per week on alternate days at the indicated number of micromoles/dose. Images represent appearance and size of typical control and SFN-treated 4 wk tumors. The values are mean \pm SEM and asterisks indicate significant change compared to control, $n=5$ mice (10 tumors), $p<0.01$. F Tumors were harvested at $4 \mathrm{wk}$ and extracts were prepared for immunoblot. Blots are shown from two representative tumors. 
3F shows immunoblots of extract prepared from two representative tumors showing that SFN treatment is associated with reduced levels of YAP1 and $\Delta \mathrm{Np} 63 \alpha$, and increased YAP1- $P$ formation. In contrast, TAZ levels are not altered by SFN treatment (Figure 3F).

\section{Role of YAP1 and SFN in HaCaT cells}

The above studies indicate that SFN reduces YAP1 and $\Delta \mathrm{Np} 63 \alpha$ level to reduce survival of SCC-13 derived ECS cells. To determine whether this is a general property shared among epidermis-derived cells, we examined SFN regulation of YAP1 and $\triangle \mathrm{Np} 63 \alpha$ in $\mathrm{HaCaT}$ cells. As shown in Figure 4A/4B/4C, SFN treatment of $\mathrm{HaCaT}$ cell-derived ECS cells reduces spheroid formation, matrigel invasion and migration. Figure 4D shows that SFN treatment reduces YAP1 and $\triangle N p 63 \alpha$. Expression of YAP(S127A) reverses SFN suppression of spheroid formation and matrigel invasion (Figure 4E/4F). Moreover, $\Delta \mathrm{Np} 63 \alpha$ overexpression reverses SFN suppression of spheroid formation (Figure 4G).

\section{Role of TAZ in response to SFN treatment}

The YAP1/TAZ transcription adaptor proteins are important controllers of cancer cell survival [9]. Our studies show that SFN treatment of cultured ECS cells (Figure 1D), or ECS cell derived tumors (Figure 3F), reduces YAP1 and $\triangle \mathrm{Np} 63 \alpha$ level, but does not alter TAZ level, suggesting that TAZ may not be a mediator of SFN action. However, we wanted to determine whether TAZ can influence the ECS cell response to SFN. Figure 5A/5B shows that TAZ knockdown reduces ECS cell spheroid formation and invasion but that loss of TAZ expression is not associated with a reduction in $\Delta \mathrm{Np} 63 \alpha$ level (Figure $5 \mathrm{C})$. Figure $5 \mathrm{D} / 5 \mathrm{E}$ shows that expression of constitutivelyactive TAZ, TAZ(S89A), reverses the SFN suppression of spheroid formation and matrigel invasion. Figure 5F demonstrates that TAZ(S89A) expression slightly increases $\Delta \mathrm{Np} 63 \alpha$ level. To determine whether this is a general effect, we assessed the role of TAZ in HaCaT cells. Figure $5 \mathrm{G} / 5 \mathrm{H}$ show that expression of TAZ(S89A) can partially reverse SFN suppression of $\mathrm{HaCaT}$ cell spheroid formation and matrigel invasion.

\section{DISCUSSION}

We recently showed that LATS1, YAP1 and $\Delta \mathrm{Np} 63 \alpha$ are part of an important ECS cell survival cascade [7]. In ECS cells, reduced LATS1 (Hippo) signaling leads to reduce YAP1 phosphorylation and enhanced nuclear accumulation of non-phosphorylated YAP1 which interacts with and stabilizes $\Delta \mathrm{Np} 63 \alpha$ to drive survival signaling [7]. $\Delta \mathrm{Np} 63 \alpha$ is a key member of the p63 family of proteins which is required for normal stem cell survival and differentiation in epidermis [13, 14]. Moreover, YAP1 and $\Delta \mathrm{Np} 63 \alpha$ have important roles in cancer [9]. We have shown that YAP1 and $\Delta \mathrm{Np} 63 \Delta$ are overexpressed in squamous cell carcinoma and the level of these prosurvival proteins is further markedly enriched in ECS cells. ECS cells comprise a small subpopulation $(0.15 \%)$ of the total tumor cell population [2] and enriched ECS cells form highly aggressive, rapidly growing and highly vascularized and invasive tumors $[2,3]$. In addition, loss of YAP1 expression leads to reduced ECS cell survival and inhibition of YAP1 function reduces tumor formation [7]. Thus, targeting YAP1 and $\Delta \mathrm{Np} 63 \alpha$ is an important potential strategy for reducing cancer stem cell survival in squamous cell carcinoma.

The idea that cancer chemoprevention agents may reduce survival of cancer stem cells is an important and evolving concept. Recent studies in colon and breast cancer suggest that diet-derived prevention agents can selectively target cancer stem cells $[33,34]$. An interesting and important observation is that cancer stem cells can be more sensitive to dietary preventive agents than nonstem cancer cells [34]. However, the mechanisms that confer this sensitivity are not well understood. SFN is an important cruciferous vegetable-derived (broccoli, etc.) cancer prevention agent [35] that has high bioavailability in vivo $[34,36]$ and displays efficacy against skin cancer in several model systems [31, 36, 37]. Moreover, it can be detected at bioactive levels in blood and tissues of broccoli-consuming human patients showing that biologically relevant levels can be achieved [31]. The concentrations of SFN used in the present studies are equivalent to levels that produce biological responses in humans.

We test the hypothesis that YAP $1 / \Delta \mathrm{Np} 63 \alpha$ signaling is targeted and suppressed by SFN as a mechanism of cancer prevention/therapy. YAP1 is resident in the nucleus where it activates cell survival signaling and proliferation. In contrast, phosphorylated YAP1 is excluded from the nucleus and subject to proteasome-mediated degradation $[9,38,39]$. Our studies show that SFN treatment increases YAP1- $P$ and reduces YAP1, and that this is associated with SFN suppression of cell spheroid formation, invasion and migration. Consistent with YAP1 functioning as a SFN target, expression of YAP(S127A), a constitutively active form of YAP1, reverses the SFN-dependent reduction in ECS cell survival, spheroid formation, matrigel invasion and migration. This suggests that YAP1 inactivation is required for SFN suppression of the ECS cell phenotype. This is consistent with recent findings suggesting that YAP1 is a key anticancer target [9, 38, 39].

Moreover, loss of YAP1 leads to a reduction in $\triangle \mathrm{Np} 63 \alpha$. This is an important event, as SFN action can be reversed by forced expression of $\Delta \mathrm{Np} 63 \alpha$ in $\mathrm{SFN}$ challenged cultures. Moreover, $\Delta \mathrm{Np} 63 \alpha$ knockdown reduces ECS cell spheroid formation, matrigel invasion and migration. This suggests that $\Delta \mathrm{Np} 63 \alpha$ is required 

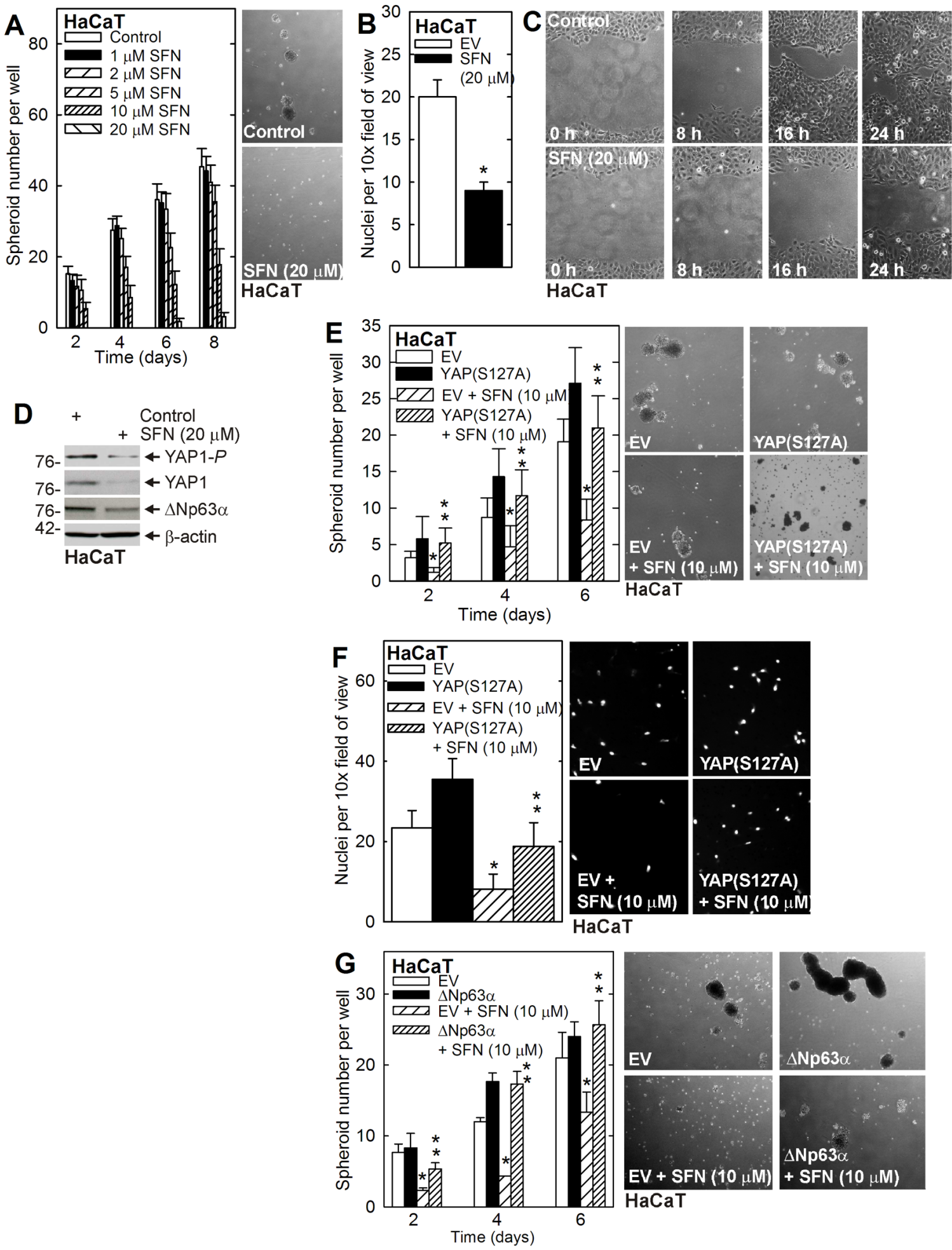

Figure 4: YAP1 and $\triangle \mathrm{Np63 \alpha}$ and the HaCaT cell response to SFN. A. B. C. HaCaT cells were seeded for spheroid formation, invasion and migration assay. Spheroids were counted and photographed at $8 \mathrm{~d}$. The values are mean \pm SEM. A significant reduction was in spheroid number was observed at 4, 6 and $8 \mathrm{~d}$ at 10 and $20 \mu \mathrm{M} \mathrm{SFN}(n=3, p<0.005)$. D. Cells were grown as spheroids for $8 \mathrm{~d}$ and then treated with $20 \mu \mathrm{M} \mathrm{SFN}$ for $48 \mathrm{~h}$ prior to collection of lysates for immunoblot. E. F. HaCaT cells were electroporated with empty vector (EV) or YAP(S127A) expression vector and at $24 \mathrm{~h}$ post-electroporation were seeded for spheroid formation and invasion assay in the presence of 0 or $20 \mu \mathrm{M}$ SFN. The image shows $6 \mathrm{~d}$ spheroids. G. HaCaT cells were electroporated as indicated and then seeded for spheroid growth assay in the presence of 0 or $10 \mu \mathrm{M}$ SFN. The images show $6 \mathrm{~d}$ spheroids. The values are mean $\pm \mathrm{SEM}$. The single asterisk indicates a significant reduction in SFN treated versus untreated control cultures. The double asterisks indicate a significant increase as compared to the SFN treated group $(n=3, p<0.005)$. 

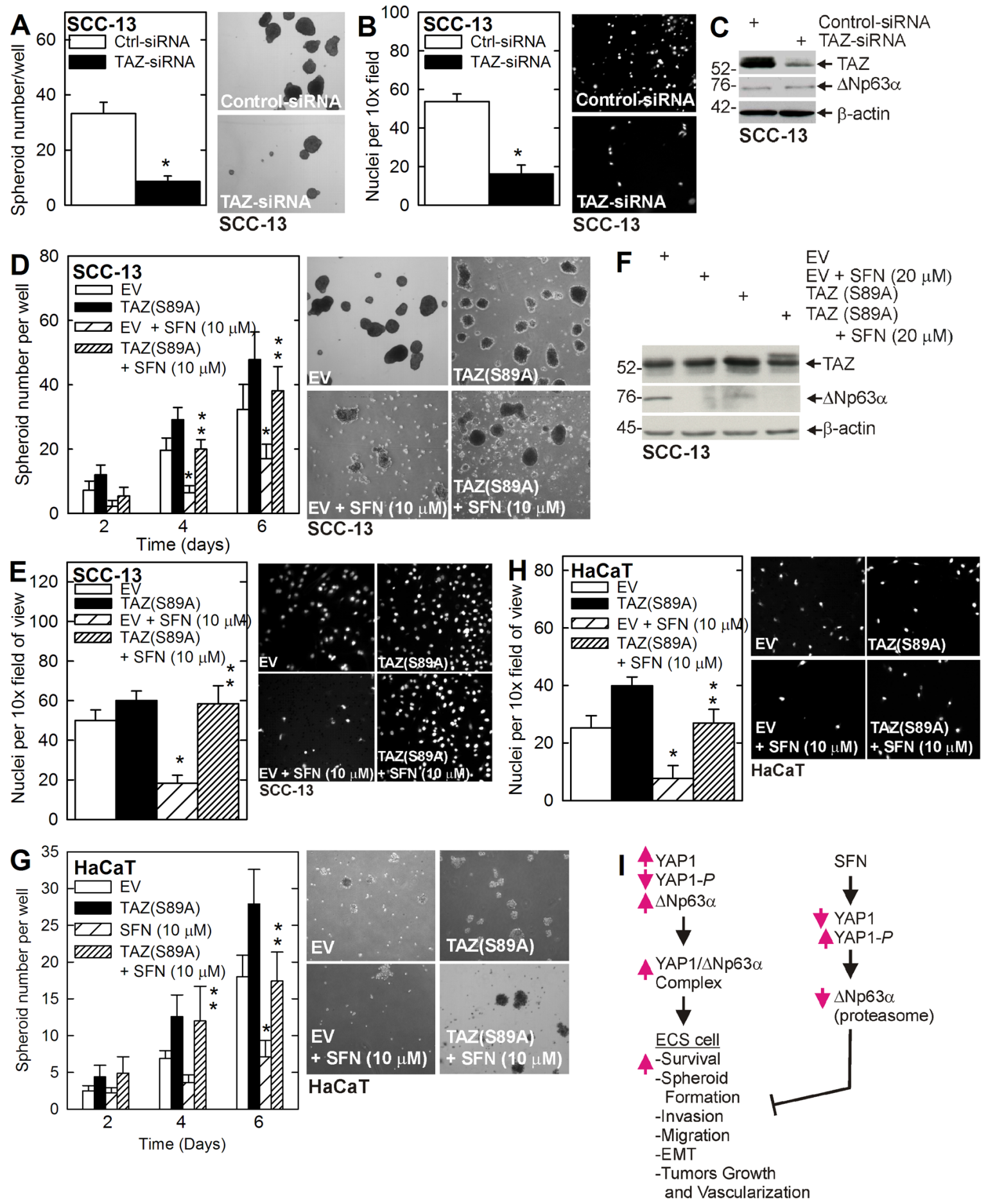

Figure 5: TAZ stimulates the ECS cell phenotype. A. B. C. ECS cells were electroporated with control- or TAZ-siRNA and then seeded for spheroid formation and $18 \mathrm{~h}$ invasion assay. Extracts for immunoblot were prepared at $48 \mathrm{~h}$ post-electroporation. The images are $6 \mathrm{~d}$ spheroids. The values are mean + SEM, and the asterisk indicates a significant reduction in SFN treated versus untreated control cultures $(n=3, p<0.005)$. D. E. F. SCC-13 cells were electroporated as indicated and the cells were seeded for spheroid formation and 18 $\mathrm{h}$ invasion assay in the presence of 0 or $10 \mu \mathrm{M} \mathrm{SFN}$. The images are of $6 \mathrm{~d}$ spheroids. The single asterisk indicates a significant reduction in SFN treated versus untreated control cultures. The double asterisks indicate a significant increase as compared to the SFN treated group ( $n$ $=3, p<0.005$ ). Extracts were prepared from $6 \mathrm{~d}$ spheroids for immunoblot. G. H. HaCaT cells were electroporated with EV or TAZ(S89A) and then seeded for spheroid and invasion assay with or without $10 \mu \mathrm{M} \mathrm{SFN}$. The asterisks indicate significance as in panels I. Model of SFN action. YAP1 levels are elevated in ECS cells where it binds to $\triangle \mathrm{Np} 63 \alpha$ leading to elevated $\Delta$ Np63 $\alpha$ levels which drives ECS cell survival, etc. SFN treatment stimulates YAP1 phosphorylation leading to reduced YAP1 level leading to proteasome-dependent loss of $\triangle \mathrm{Np} 63 \alpha$ which results in reduced ECS cell survival and reduced tumor growth. 
for YAP1-induced survival in ECS cells. To understand the mechanism of regulation of $\Delta \mathrm{Np} 63 \alpha$ level by YAP1, ECS cells were treated with SFN and $\triangle \mathrm{Np} 63 \alpha$ mRNA and protein levels were monitored. SFN treatment does not impact $\Delta \mathrm{Np} 63 \alpha \mathrm{mRNA}$ level, but a large drop in $\Delta \mathrm{Np} 63 \alpha$ level is observed. This suggests that the regulation is not at the level of transcription or mRNA stability. Treatment of ECS cells with SFN in the presence of proteasome inhibitor restores $\Delta \mathrm{Np} 63 \alpha$ expression. This suggests a model in which YAP $1 / \Delta \mathrm{Np} 63 \alpha$ interaction stabilizes nuclear $\Delta \mathrm{Np} 63 \alpha$ leading to enhanced survival signaling.

To assess whether loss of YAP $1 / \Delta \mathrm{Np} 63 \alpha$ is a common response to SFN treatment, we examined the impact of SFN treatment in HaCaT cell-derived ECS cells. $\mathrm{HaCaT}$ cells are an immortalized line of epidermis-derived keratinocytes [40]. The HaCaT studies confirm that SFN treatment reduces spheroid formation, matrigel invasion and migration, and show that this is associated with reduced YAP1 and reduced $\triangle \mathrm{Np} 63 \alpha$. Moreover, forced expression of $\mathrm{YAP}(\mathrm{S} 127 \mathrm{~A})$ or $\triangle \mathrm{Np} 63 \alpha$ in SFN treated HaCaT-derived ECS cells, protects the cells against SFN and restores ECS cell spheroid formation, and matrigel invasion. Thus, SFN regulation of YAP1 and $\Delta \mathrm{Np} 63 \alpha$ is observed in multiple epidermis-derived cell types.

To assess the role of these signaling proteins during tumor formation and response to SFN, we treated ECS cell tumor xenografts with SFN and monitored the impact on YAP $1 / \Delta N p 63 \alpha$ level. These studies show that $\mathrm{SFN}$ reduces ECS cell tumor formation and that this is associated with increased YAP1-P, reduced total YAP1 and reduced $\triangle \mathrm{Np} 63 \alpha$. These findings suggest that the SFN-stimulated signaling changes observed in cultured cells are also observed in SFN treated tumors in vivo.

YAP1 often interacts with TAZ to modulate transcription [38, 39]. Our previous study shows that YAP1 is an important mediator of ECS cell survival, but that TAZ is not required [7]. Indeed, our present study shows that SFN treatment does not reduced TAZ level in cultured cells or in SFN-treated tumors, suggesting that TAZ does not play a major role in the response to SFN. However, we wanted to determine whether TAZ can influence ECS cell function. These studies reveal that TAZ loss reduces spheroid formation and matrigel invasion, but that loss of TAZ is not associated with loss of $\triangle N p 63 \alpha$. Moreover, forced expression of TAZ(S89A), a constitutively activate form of TAZ, antagonizes SFN action and restores spheroid formation and matrigel invasion. We conclude that although TAZ does not appear to play a role in SFN suppression of the ECS cell phenotype, TAZ can independently impact ECS cell survival and resistance to SFN. Additional studies will be necessary to further understand the mechanism of TAZ action in this context.

Based on these studies we propose that ECS cell survival is associated with elevated YAP1 (and reduced YAP1-P) leading to YAP1 association of with and stabilization of $\Delta \mathrm{Np} 63 \alpha$, and that $\Delta \mathrm{Np} 63 \alpha$ then drives an increase in ECS cell survival (Figure 5I). We further propose that SFN treatment increases YAP1- $P$ and reduces YAP1 level, and that loss of YAP1 leads to proteolytic degradation of $\triangle \mathrm{Np} 63 \alpha$ to reduce ECS cell survival and growth. Our observations suggest that this mechanism exists in multiple cell types and in tumors, and that this mechanism is worthy of additional study as a potentially important mechanism of SFN-mediated cancer prevention and therapy.

\section{MATERIALS AND METHODS}

\section{Antibodies and reagents}

Sodium pyruvate (11360-070), DMEM (11960$077), 0.25 \%$ trypsin-EDTA (25200-056) and L-Glutamine (25030-164) were purchased from Gibco (Grand Island, NY). Heat-inactivated fetal calf serum (FCS, F4135), lactacystin (L6785) and anti- $\beta$-actin (A5441) were purchased from Sigma (St. Louis, Mo). Cell lysis Buffer (9803) was purchased from Cell Signaling Technology (Danvers, MA). YAP1 (4912), YAP1-P (13008), Histone 3 (\#9717) and TAZ (4883) antibodies were purchased from Cell Signaling Technologies. Anti-p63 (sc-8431), anti-ubiquitin (sc-9133) and anti-TAZ-P (17610) were purchased from Santa Cruz. Anti-pan-TEAD (ab1791) was purchased from Abcam (Cambridge, MA). Peroxidaseconjugated anti-mouse IgG (NXA931) and anti-rabbit IgG (NA934V) were obtained from GE healthcare (Buckinghamshire, UK). DAPI (D9542) was purchased from Sigma Chemicals (St. Louis, MO). p63- (sc-36161), TAZ- (sc-38568A) and control-siRNA (sc-37007) were purchased from Santa Cruz (Dallas, TX). YAP1-siRNA (S102662954) was purchased from Qiagen (Valencia, CA). Matrigel (354234) and BD Biocoat cell inserts (353097) were purchased from BD Biosciences (Franklin Lakes, NJ). Sulforaphane (S8044) was purchased from LKT Laboratories INC (St. Paul, MN). YAP(S127A) (Addgene plasmid \# 27370) and TAZ(S89A) (Addgene plasmid \# 32840) were from Kunliang Guan [41].

\section{Immunoblot}

Cell extracts were prepared in lysis buffer, and equivalent amounts of protein were electrophoresed on denaturing and reducing $10 \%$ polyacrylamide gels and transferred to nitrocellulose membrane. Membranes were blocked with $5 \%$ nonfat dry milk for one hour and incubated in 5\% nonfat dry milk with containing 1:1000 diluted primary antibody. Blots were washed and then incubated with secondary antibody (1:5000) for $2 \mathrm{~h}$. Secondary antibody binding was visualized using ECL (Amersham) chemiluminescence detection technology. 


\section{Spheroid formation assay}

Cancer cells were maintained under attached conditions in growth media containing DMEM (Invitrogen, Frederick, MD) supplemented with $4.5 \mathrm{mg} / \mathrm{ml}$ D-glucose, $2 \mu \mathrm{M}$ L-glutamine, $100 \mathrm{mM}$ sodium pyruvate, and $5 \%$ fetal calf serum. For spheroid formation, nearconfluent monolayer cultures are dissociated with $0.25 \%$ trypsin, followed by serum-dependent trypsin inactivation. The cells are collected by centrifugation, and resuspended in spheroid media, consisting of DMEM/F12 (1:1) (DMT10-090-CV, Mediatech INC, Manassa, VA) containing 2\% B27 serum-free supplement (17504-044, Invitrogen, Frederick, MD), 20 ng/ml EGF (E4269, Sigma, St. Louis), $0.4 \%$ bovine serum albumin (B4287, Sigma) and $4 \mu \mathrm{g} / \mathrm{ml}$ insulin (19278 Sigma, St. Louis, MO.), and plated at 40,000 cells per $9.6 \mathrm{~cm}^{2}$ well in six well ultralow attachment Costar cluster dishes (4371, Corning, Tewksbury, MA).

\section{Cell fractionation studies}

The cells used for this experiment were day 8 spheroids that were treated with 0 or $20 \mu \mathrm{M}$ SFN for $48 \mathrm{~h}$ before extract preparation. The NE-PER Nuclear and Cytoplasmic Extraction Kit (product \# 78833) was obtained from Thermo Scientific (Waltham, MA). For total extracts, $40 \mu \mathrm{g}$ of protein was electrophoresed and equal numbers of cell equivalents were loaded in lanes comparing the cytosol and nuclear fractions.

\section{qRT-PCR}

Total RNA was isolated using the RNAspin Mini Kit (GE Healthcare) and reverse transcribed using the Superscript III reverse transcriptase (Invitrogen, Carlsbad, CA). RNA $(1 \mu \mathrm{g})$ was used for cDNA preparation. The Light Cycler 480 SYBR Green I Master mix (Roche Diagnostics) was used to measure mRNA level. $\Delta$ Np63a mRNAlevel was detected and signals were normalized to the level of cyclophilin A mRNA. The following gene specific primers were used for detection of mRNA levels: $\triangle \mathrm{Np} 63 \alpha$ (forward: 5'-5'-GGA AAA CAA TGC CCA GAC TCA, reverse: 5'-5'-TGT TCA GGA GCC CCA GGT T) and cyclophilin A (forward: 5'-CAT CTG CAC TGC CAA GAC TGA, reverse: 5'-TTC ATG CCT TCT TTC ACT TTGC).

\section{Electroporation of nucleic acids}

Cancer cells $(150,000)$ were plated in $60 \mathrm{~mm}$ plates in growth media. After $24 \mathrm{~h}$, when approximately $50 \%$ confluent, the cells were collected using $0.25 \%$ trypsin, centrifuged at $200 \mathrm{x} \mathrm{g}$, washed with sterile phosphate-buffered saline $(\mathrm{pH} 7.5)$, and suspended in $100 \mu \mathrm{l}$ of keratinocyte nucleofection reagent VPD-1002 (Walkersville, MD) for electroporation with siRNA or plasmid. The cell suspension, contained either 3 $\mu \mathrm{g}$ of siRNA or $2 \mu \mathrm{g}$ of plasmid was gently mixed and electroporated using the T- 018 setting on the AMAXA Electroporator. Immediately after electroporation, prewarmed spheroid media was added and the suspension was transferred to monolayer culture. After $24 \mathrm{~h}$, the cells were harvested and plated for spheroid formation, migration and invasion assays. For siRNA experiments, the cells were harvested and electroporated a second time, following the same protocol, $72 \mathrm{~h}$ after the initial electroporation.

\section{Invasion and migration assays}

Matrigel (BD Biolabs) was diluted in $0.01 \mathrm{M}$ Tris$\mathrm{HCl} / 0.7 \% \mathrm{NaCl}$ and filter sterilized and $0.1 \mathrm{ml}$ was used to cover BD BioCoat cell inserts. After $2 \mathrm{~h}$, cells were harvested and 25,000 cells were plated in $100 \mu \mathrm{l}$ of growth medium containing 1\% FCS on top of the Matrigel. Growth medium containing 10\% FCS was added to the lower well chamber and the cells were incubated overnight at $37 \mathrm{C}$. The following day, excess cells from the top side of the membrane were removed with a cotton swab, and the membrane was rinsed with phosphate buffered saline, fixed with $4 \%$ paraformaldehyde for $10 \mathrm{~min}$, washed, and stained in $1 \mu \mathrm{g} / \mathrm{ml}$ DAPI for $10 \mathrm{~min}$. The underside of the membrane was photographed with an inverted fluorescent microscope to count the number of nuclei. For migration, SCC-13 cells ( 2 million) were plated on $10 \mathrm{~cm}$ dishes in spheroid media under monolayer conditions and allowed to attach overnight. Once confluent, a $10 \mu \mathrm{l}$ pipette was used to create scratch wounds. The dishes were washed with phosphate buffered saline to remove the dislodged cells and fresh spheroid medium was added with or without SFN. Images were taken at 10x to monitor cell migration into the wounded area.

\section{Tumor xenograft assays}

Spheroid-derived cancer cells were prepared as a single cell suspension by trypsin digestion, resuspended in phosphate buffered saline containing 30\% Matrigel and $100 \mu \mathrm{l}$ containing $0.5 \times 10^{6}$ cells was injected subcutaneously in the two front flanks of NOD scid IL2 receptor gamma chain knockout mice (NSG mice) using a 26.5 gauge needle. Five mice were used per group (two tumors per mouse). SFN was dissolved in sterile saline and delivered by oral gavage at 20 micromoles per dose in $100 \mu \mathrm{L}$ on alternate days (M/W/F). Tumor growth was monitored by measuring tumor diameter and calculating tumor volume using the formula, volume $=$ $4 / 3 \pi \times(\text { diameter } / 2)^{3}$. Mice were euthanized by injection of $250 \mu \mathrm{l}$ of a $2.5 \%$ stock of Avertin per mouse followed 
by cervical dislocation of the neck. Tumor samples were harvested to prepare extracts for immunoblot and sections for immunostaining. These experiments were reviewed and approved by the University of Maryland-Baltimore Institutional Animal Care and Use Committee.

\section{ACKNOWLEDGMENTS}

This work was supported by grants from the National Institutes of Health to RLE (R01 CA131074 and R01 CA184027).

\section{CONFLICTS OF INTEREST}

The authors declare no conflict of interest

\section{REFERENCES}

1. Lansbury L, Bath-Hextall F, Perkins W, Stanton W, Leonardi-Bee J. Interventions for non-metastatic squamous cell carcinoma of the skin: systematic review and pooled analysis of observational studies. BMJ. 2013; 347:f6153.

2. Adhikary G, Grun D, Kerr C, Balasubramanian S, Rorke EA, Vemuri M, Boucher S, Bickenbach JR, Hornyak T, Xu W, Fisher ML, Eckert RL. Identification of a population of epidermal squamous cell carcinoma cells with enhanced potential for tumor formation. PLoS One. 2013; 8:e84324.

3. Grun D, Adhikary G, Eckert RL. VEGF-A acts via neuropilin-1 to enhance epidermal cancer stem cell survival and formation of aggressive and highly vascularized tumors. Oncogene. 2016; 35:4379-87.

4. Adhikary G, Grun D, Balasubramanian S, Kerr C, Huang JM, Eckert RL. Survival of skin cancer stem cells requires the Ezh2 polycomb group protein. Carcinogenesis. 2015; 36:800-10.

5. Eckert RL, Fisher ML, Grun D, Adhikary G, Xu W, Kerr C. Transglutaminase is a tumor cell and cancer stem cell survival factor. Mol Carcinog. 2015; 54:947-58.

6. Fisher ML, Adhikary G, Grun D, Kaetzel DM, Eckert RL. The Ezh2 polycomb group protein drives an aggressive phenotype in melanoma cancer stem cells and is a target of diet derived sulforaphane. Mol Carcinog. 2016; 55:2024 36.

7. Fisher ML, Kerr C, Adhikary G, Grun D, Xu W, Keillor JW, Eckert RL. Transglutaminase interaction with a6/ b4-integrin to stimulates YAP1-dependent DNp63a stabilization and leads to enhanced cancer stem cell survival and tumor formation. Cancer Res. 2016; 76:7265-76.

8. Barry ER, Camargo FD. The Hippo superhighway: signaling crossroads converging on the Hippo/Yap pathway in stem cells and development. Curr Opin Cell Biol. 2013; 25:247-53.

9. Harvey KF, Zhang X, Thomas DM. The Hippo pathway and human cancer. Nat Rev Cancer. 2013; 13:246-57.
10. Ramos A, Camargo FD. The Hippo signaling pathway and stem cell biology. Trends Cell Biol. 2012; 22:339-46.

11. Zhao B, Tumaneng K, Guan KL. The Hippo pathway in organ size control, tissue regeneration and stem cell selfrenewal. Nat Cell Biol. 2011; 13:877-83.

12. Zhang H, Pasolli HA, Fuchs E. Yes-associated protein (YAP) transcriptional coactivator functions in balancing growth and differentiation in skin. Proc Natl Acad Sci USA. $2011 ; 108: 2270-75$.

13 Koster MI, Huntzinger KA, Roop DR. Epidermal differentiation: transgenic/knockout mouse models reveal genes involved in stem cell fate decisions and commitment to differentiation. J Investig Dermatol Symp Proc. 2002; $7: 41-45$.

14. Senoo M, Pinto F, Crum CP, McKeon F. p63 Is essential for the proliferative potential of stem cells in stratified epithelia. Cell. 2007; 129: 523-536.

15. Mills AA, Zheng B, Wang XJ, Vogel H, Roop DR, Bradley A. p63 is a p53 homologue required for limb and epidermal morphogenesis. Nature. 1999; 398:708-13.

16. Koster MI, Kim S, Mills AA, DeMayo FJ, Roop DR. p63 is the molecular switch for initiation of an epithelial stratification program. Genes Dev. 2004; 18:126-31.

17. Yang A, Kaghad M, Wang Y, Gillett E, Fleming MD, Dotsch V, Andrews NC, Caput D, McKeon F. p63, a p53 homolog at 3q27-29, encodes multiple products with transactivating, death-inducing, and dominant-negative activities. Mol Cell. 1998; 2:305-16.

18. Reis-Filho JS, Torio B, Albergaria A, Schmitt FC. p63 expression in normal skin and usual cutaneous carcinomas. J Cutan Pathol. 2002; 29:517-23.

19. Clarke JD, Dashwood RH, Ho E. Multi-targeted prevention of cancer by sulforaphane. Cancer Lett. 2008; 269:291-304.

20. Egner PA, Chen JG, Zarth AT, Ng DK, Wang JB, Kensler KH, Jacobson LP, Munoz A, Johnson JL, Groopman JD, Fahey JW, Talalay P, Zhu J et al. Rapid and sustainable detoxication of airborne pollutants by broccoli sprout beverage: results of a randomized clinical trial in China. Cancer Prev Res (Phila). 2014; 7:813-23.

21. Ho E, Clarke JD, Dashwood RH. Dietary sulforaphane, a histone deacetylase inhibitor for cancer prevention. J Nutr. 2009; 139:2393-96.

22. Singh SV, Warin R, Xiao D, Powolny AA, Stan SD, Arlotti JA, Zeng Y, Hahm ER, Marynowski SW, Bommareddy A, Desai D, Amin S, Parise RA et al. Sulforaphane inhibits prostate carcinogenesis and pulmonary metastasis in TRAMP mice in association with increased cytotoxicity of natural killer cells. Cancer Res. 2009; 69:2117-25.

23. Li Y, Zhang T, Li X, Zou P, Schwartz SJ, Sun D. Kinetics of sulforaphane in mice after consumption of sulforaphaneenriched broccoli sprout preparation. Mol Nutr Food Res. 2013; 57:2128-36.

24. Clarke JD, Hsu A, Yu Z, Dashwood RH, Ho E. Differential effects of sulforaphane on histone deacetylases, cell 
cycle arrest and apoptosis in normal prostate cells versus hyperplastic and cancerous prostate cells. Mol Nutr Food Res. 2011; 55:999-1009.

25. Dashwood RH, Ho E. Dietary agents as histone deacetylase inhibitors: sulforaphane and structurally related isothiocyanates. Nutr Rev. 2008; 66:S36-38.

26. Singh AV, Xiao D, Lew KL, Dhir R, Singh SV. Sulforaphane induces caspase-mediated apoptosis in cultured PC-3 human prostate cancer cells and retards growth of PC-3 xenografts in vivo. Carcinogenesis. 2004; 25:83-90.

27. Vyas AR, Hahm ER, Arlotti JA, Watkins S, Stolz DB, Desai D, Amin S, Singh SV. Chemoprevention of prostate cancer by d,l-sulforaphane is augmented by pharmacological inhibition of autophagy. Cancer Res. 2013; 73:5985-95.

28. Kensler TW, Ng D, Carmella SG, Chen M, Jacobson LP, Munoz A, Egner PA, Chen JG, Qian GS, Chen TY, Fahey JW, Talalay P, Groopman JD et al. Modulation of the metabolism of airborne pollutants by glucoraphanin-rich and sulforaphane-rich broccoli sprout beverages in Qidong, China. Carcinogenesis. 2012; 33:101-07.

29. Cheung KL, Kong AN. Molecular targets of dietary phenethyl isothiocyanate and sulforaphane for cancer chemoprevention. AAPS J. 2010; 12:87-97.

30. Su ZY, Zhang C, Lee JH, Shu L, Wu TY, Khor TO, Conney AH, Lu YP, Kong AN. Requirement and epigenetics reprogramming of $\mathrm{Nrf} 2$ in suppression of tumor promoter TPA-induced mouse skin cell transformation by sulforaphane. Cancer Prev Res (Phila). 2014; 7:319-29.

31. Xu C, Huang MT, Shen G, Yuan X, Lin W, Khor TO, Conney AH, Kong AN. Inhibition of 7,12-dimethylbenz(a) anthracene-induced skin tumorigenesis in C57BL/6 mice by sulforaphane is mediated by nuclear factor E2-related factor 2. Cancer Res. 2006; 66:8293-96.

32. Balasubramanian S, Chew YC, Eckert RL. Sulforaphane Suppresses Polycomb Group Protein Level via a
Proteasome-Dependent Mechanism in Skin Cancer Cells. Mol Pharmacol. 2011; 80:870-78.

33. Kim NG, Gumbiner BM. Adhesion to fibronectin regulates Hippo signaling via the FAK-Src-PI3K pathway. J Cell Biol. 2015; 210:503-15.

34. Li Y, Zhang T, Korkaya H, Liu S, Lee HF, Newman B, Yu Y, Clouthier SG, Schwartz SJ, Wicha MS, Sun D. Sulforaphane, a dietary component of broccoli/broccoli sprouts, inhibits breast cancer stem cells. Clin Cancer Res. 2010; 16:2580-90.

35. Subramaniam D, Ramalingam S, Houchen CW, Anant S. Cancer stem cells: a novel paradigm for cancer prevention and treatment. Mini Rev Med Chem. 2010; 10:359-71.

36. Dinkova-Kostova AT, Jenkins SN, Fahey JW, Ye L, Wehage SL, Liby KT, Stephenson KK, Wade KL, Talalay P. Protection against UV-light-induced skin carcinogenesis in SKH-1 high-risk mice by sulforaphane-containing broccoli sprout extracts. Cancer Lett. 2006; 240:243-52.

37. Fimognari C, Hrelia P. Sulforaphane as a promising molecule for fighting cancer. Mutat Res. 2007; 635:90-104.

38. Kodaka M, Hata Y. The mammalian Hippo pathway: regulation and function of YAP1 and TAZ. Cell Mol Life Sci. 2015; 72:285-306.

39. Halder G, Johnson RL. Hippo signaling: growth control and beyond. Development. 2011; 138:9-22.

40. Boukamp P, Petrussevska RT, Breitkreutz D, Hornung J, Markham A, Fusenig NE. Normal keratinization in a spontaneously immortalized aneuploid human keratinocyte cell line. J Cell Biol. 1988; 106:761-71.

41. Lei QY, Zhang H, Zhao B, Zha ZY, Bai F, Pei XH, Zhao S, Xiong Y, Guan KL. TAZ promotes cell proliferation and epithelial-mesenchymal transition and is inhibited by the hippo pathway. Mol Cell Biol. 2008; 28:2426-36. 